\title{
Seuche Cannabis? Kritische Bemerkungen zu neueren epidemiologischen Studien
}

\author{
J. Kalke \\ U. Verthein \\ H. Stöver
}

\section{Is Cannabis an Epidemic? Critical Remarks to Recent Epidemiological Studies}

\section{Zusammenfassung}

In der öffentlichen Berichterstattung wurde in der letzten Zeit auf eine dramatische Zunahme problematischen Cannabis-Konsums unter jungen Menschen hingewiesen. Lässt sich dies aber tatsächlich mit empirischen Zahlen belegen? In diesem Beitrag werden aktuelle epidemiologische Untersuchungen unter die „kritische Lupe“ genommen und in empirischer sowie methodischer Hinsicht auf ihre Aussagekraft hin überprüft. Dazu gehören u.a. die Untersuchung zur Drogenaffinität Jugendlicher in der Bundesrepublik Deutschland der BZgA, die Repräsentativerhebung zum Gebrauch und Missbrauch psychoaktiver Substanzen bei Erwachsenen und die Deutsche Suchthilfestatistik. Darüber hinaus werden auch Ergebnisse aus regionalen Erhebungen präsentiert. Werden die Ergebnisse aller hier analysierten Untersuchungen zusammen betrachtet, lässt sich zumindest der empirisch gesicherte Schluss ziehen, dass es in den letzten 10 Jahren zu keinem Rückgang des Cannabis-Konsums in Deutschland gekommen ist. Ob er hingegen zugenommen hat, und wenn ja in welchem Ausmaß, kann aufgrund der teilweise widersprüchlichen Ergebnisse und der teilweise kritikwürdigen Erhebungsinstrumente der Untersuchungen nicht eindeutig festgestellt werden. Nach den ermittelten Prävalenzen der letzten BZgA-Studie ist der Cannabis-Konsum bei den jungen Erwachsenen seit 1997 stabil, nach den Ergebnissen der aktuellen Repräsentativerhebung hat er seitdem in dieser Altersgruppe zugenommen. Auch die Ergebnisse von regionalen Erhebungen lassen keinen eindeutigen Trend erkennen. Das Gleiche gilt für vorliegende Zahlen aus anderen europäischen Staaten.

Schliuisselwörter

Cannabis · epidemiologische Studien · Prävalenzen

\section{Abstract}

Dramatic increases of problematic cannabis use among young people have recently been reported by the media. Is this really based on empirical data? The present article critically examines recent epidemiological studies in terms of their empirical and methodological meaningfulness. The evaluation includes e.g. the investigation of drug affinity among young people in the Federal Republic of Germany by the BZgA, the epidemiological survey of substance abuse among adults in Germany and the German statistical report on outpatient treatment. In addition, results of regional surveys will be presented. When summarising the results of all analysed investigations, we come at least to the empirically-based conclusion that cannabis use did not decrease in Germany in the last ten years. However, the partly controversial results and the survey methods that partly justify critique do not allow to draw any conclusions as to whether cannabis use has increased and, if so, to what extent. According to the prevalence found by the BzgA study, cannabis use among young adults did not change since 1997; the epidemiological survey found that it did increase. Results of regional surveys do not show a clear trend either, nor do results from other European countries.

Key words

Cannabis · epidemiological studies $\cdot$ prevalences 
Einleitung

Im Juni letzten Jahres machte „Der Spiegel“ mit einer ABC-Anfängerin auf, die als Schultüte einen überdimensionalen Joint trug: „Die Seuche Cannabis“. Der „Fokus“ zog einige Monate später nach und berichtete über den „Drogenbrennpunkt Schule“. Diese Ausgaben der beiden auflagenstärksten Nachrichtenmagazine waren der Höhepunkt einer öffentlichen Diskussion über die vermeintlichen oder tatsächlichen Gefahren des Cannabis-Konsums. In der Berichterstattung wurden überwiegend klinische Fälle von cannabisabhängigen Jugendlichen - teilweise sogar Kindern - als Hinweise auf vermeintliche epidemiologische Entwicklungen präsentiert und auf diese Weise versucht, eine dramatische Zunahme des Cannabis-Konsums unter jungen Menschen zu belegen. Auch der kritische Hinweis auf die „68er-Generation“ fehlte nicht, die das Kiffen der eigenen Kinder ignorieren oder zumindest verniedlichen würde.

Ebenso nahmen sich Fachzeitschriften des Themas an: „Sucht“ gewann Karl-Ludwig Täschner als Gastherausgeber für einen Themenschwerpunkt Cannabis. Er erneuerte im Editorial seine bekannte These, dass regelmäßiger Cannabis-Konsum verantwortlich für Psychosen und das so genannte amotivationale Syndrom sei [1].

Die Politik konnte sich diesen Hiobsbotschaften nicht entziehen: Parteiübergreifend wurde dringender Handlungsbedarf gesehen. Eine im November 2004 vom Bundesministerium für Gesundheit und Soziale Sicherung (BMGS) ausgerichtete Cannabis-Fachkonferenz wurde von der Drogenbeauftragten der Bundesregierung mit der Überschrift „Risiken werden oft verharmlost“ zusammengefasst, weil es Hinweise gäbe, dass immer mehr junge Leute in exzessivem Ausmaß kifften und praktisch den ganzen Tag „breit“ seien [2]. Verwiesen wurde auf einen europaweiten Trend der Zunahme des Cannabis-Konsums sowie auf die Verringerung des Einstiegsalters von Erstkonsumenten.

Von den auf der Konferenz versammelten Experten ${ }^{1}$ wurden Handlungsempfehlungen verabschiedet, in denen eine sachliche und ideologiefreie Diskussion über das Thema Cannabis gefordert wird. Gleichzeitig wurde aber die generelle Feststellung getroffen, dass Cannabis-Konsum immer mit Risiken verbunden sei [2]. Außerdem konstatierten die Experten eine teilweise besorgniserregende Unkenntnis über das Risikopotenzial von Cannabis bei Jugendlichen.

Scheinbar gestützt werden die Aussagen von den neuesten Zahlen zur Verbreitung legaler und illegaler Drogen unter Jugendlichen und jungen Erwachsenen in Deutschland, die von der Bundeszentrale für gesundheitliche Aufklärung (BZgA) veröffentlicht wurden. Danach habe die Akzeptanz für Cannabis unter Jugendlichen deutlich zugenommen und es gäbe einen entsprechenden Anstieg des Cannabis-Konsums in der jüngeren Bevölkerung, so der Inhalt einer begleitenden Presseveröffentlichung [3].

\footnotetext{
1 Aus redaktionellen Gründen wird im Folgenden immer nur die männliche Ausdruckweise gewählt (Experten etc.). Das schließt natürlich immer auch die weiblichen Personen (Expertinnen) mit ein.
}

Die neuen epidemiologischen Entwicklungen, Problemzuschreibungen und fachlichen Stellungnahmen haben die cannabispolitische Landschaft in Deutschland deutlich beeinflusst: Während sich Mitte der 90er Jahre noch die meisten Gesundheitsminister der Bundesländer infolge des Bundesverfassungsgerichtsurteils von 1994 für einen Modellversuch zur kontrollierten Abgabe von Cannabis einsetzten, sind heute offensichtlich - nicht einmal mehr ergebnisoffene Risikodiskurse erwünscht. So heißt es im jüngsten Koalitionsvertrag von CDU und SPD in Schleswig-Holstein: „Wir werden eine öffentliche Risikodebatte zu Cannabis führen, die nicht auf eine Legalisierung abzielt [4].“ In einem solchen Diskussionsklima haben es sogar Veröffentlichungen anerkannter Institutionen, wie z. B. das Cannabis-Buch der Deutschen Hauptstelle für Suchtfragen, in dem man sich um Vielfalt sowie Differenzierung und Ergebnisoffenheit des Risikodiskurses bemüht, schwer [5].

Lässt sich nun aber tatsächlich mit den aktuellen Untersuchungen das Auftreten einer „Cannabis-Epidemie“ belegen? In diesem Beitrag sollen die aktuell bedeutsamsten epidemiologischen Untersuchungen der letzten Zeit unter die „kritische Lupe“ genommen und in empirischer sowie methodischer Hinsicht auf ihre Aussagekraft und Belastbarkeit hin überprüft werden.

Im Mittelpunkt stehen die Untersuchung zur Drogenaffinität Jugendlicher in der Bundesrepublik Deutschland [6], die Repräsentativerhebung zum Gebrauch und Missbrauch psychoaktiver Substanzen bei Erwachsenen in Deutschland [7] und die Deutsche Suchthilfestatistik [8]. Zusätzlich werden auch Ergebnisse aus einigen - weniger beachteten - regionalen Erhebungen präsentiert $[10,12,13]$.

Es sei betont, dass es nicht der Anspruch dieses Beitrages ist, ein systematisches Review über alle nationalen oder europäischen epidemiologischen Erhebungen der letzten Jahre zu geben. Für diesen Beitrag wurden vor allem die Untersuchungen zur Beurteilung ausgewählt, die in der politischen und fachöffentlichen Diskussion der letzten Zeit in Deutschland eine wichtige Rolle gespielt haben. ${ }^{2}$

\section{Drogenaffinitätsstudie 2004}

Die neuesten Daten zum Ausmaß des Cannabis-Konsums bei Jugendlichen und jungen Erwachsenen liefert die im November 2004 veröffentlichte so genannte Drogenaffinitätsstudie der BZgA [6]. Die Ergebnisse beruhen auf den Angaben einer Zufallsstichprobe von 3000 Personen im Alter zwischen 12 und 25 Jahren. In den Monaten Januar und Februar 2004 wurden computergestützte Telefoninterviews durchgeführt.

Bei den Drogenaffinitätsstudien handelt es sich um repräsentative Wiederholungsbefragungen, die in mehrjährigen Abständen

\footnotetext{
2 Die HBSC-Studie und ihre nationalen oder sogar bundesländerspezifischen Auswertungen wurden außer Acht gelassen, weil sie keine Trendzahlen enthalten. HBSC: Health Behaviour in School-aged Chil-
} dren Study (www.hbsc.org). 
durchgeführt werden, um u. a. langfristige Trends im Drogenkonsumverhalten sowie die Einstellung und Konsumbereitschaft junger Leute gegenüber Drogen abbilden zu können. Wenngleich sich der überwiegende Teil der Affinitätsstudie eingehenden Analysen zu Probierbereitschaft, Motiven, Einstellungen und Gesundheitsbewusstsein widmet, erfahren die Angaben zur Prävalenz des Drogengebrauchs - gerade von politischer Seite - in der Regel besondere Aufmerksamkeit.

Betrachtet man die Lifetime-Prävalenzen aus dem Jahr 2004, so wird deutlich, dass $31 \%$ der Jugendlichen und jungen Erwachsenen zumindest einmal in ihrem Leben Cannabis konsumiert haben (illegale Drogen insgesamt: $32 \%$ ). Da generell die Wahrscheinlichkeit für jeglichen Konsum mit zunehmendem Alter steigt, ergeben sich hier deutliche Altersunterschiede: In der Gruppe der 12 - bis 15 -Jährigen waren es $7 \%$, bei den 16 - bis 19-Jährigen 36\% und unter den jungen Erwachsenen (20-25 Jahre) bereits $44 \%$, die bisher Cannabis zumindest einmal probiert haben. Seit 1997 sind die Lifetime-Prävalenzen illegalen Drogenkonsums (der praktisch immer gleichbedeutend ist mit Cannabis-Konsum) von $23 \%$ auf heute $32 \%$ gestiegen. Dies nahm die Drogenbeauftragte der Bundesregierung zum Anlass, um von einem „Jugendkult Cannabis“ zu sprechen. Fraglich ist jedoch, ob solche Angaben zur Lifetime-Prävalenz überhaupt eine geeignete Basis für die Beschreibung des Ausmaßes des Cannabis-Konsums unter Jugendlichen darstellen, sagen sie doch lediglich aus, ob überhaupt einmal zur Droge Cannabis gegriffen wurde, unabhängig davon, wann und wie oft dies geschehen ist. Dies gilt umso mehr, da von den $32 \%$ Drogenerfahrenen knapp die Hälfte (44\%) die Substanz (in der Regel Cannabis) nur einoder zweimal in ihrem Leben probiert hat.

Bedeutsamer für die aktuelle Situation zum Cannabis-Konsum unter Jugendlichen sind die Zahlen zur 12-Monats-Prävalenz und zum gegenwärtigen Gebrauch. Nach den Ergebnissen der neuesten Drogenaffinitätsstudie haben $13 \%$ der 12 - bis 25 -Jährigen in den letzten 12 Monaten Cannabis genommen, 5\% der Jugendlichen und jungen Erwachsenen bezeichnen sich selbst als „gegenwärtige“ Cannabis-Konsumenten. Folgt man der in der Studie gegebenen Definition des regelmäßigen Konsums, so kommt man auf 3\% der Befragten, die in den letzten 12 Monaten häufiger als 10-mal Cannabis genommen haben.

Zudem wird deutlich, dass der aktuelle Drogenkonsum unter den Jugendlichen seit 1997 weitgehend stabil geblieben ist. So betrug die 12-Monats-Prävalenz 1997 12\% und in den Jahren 2001 und 2004 13\%. Ein leichter Anstieg ist allerdings bei den männlichen Jugendlichen (von 14\% in 1997 über 15\% in 2001 auf $17 \%$ in 2004) sowie in der Gruppe der 16- bis 19-Jährigen zu beobachten. Demgegenüber blieben die Prävalenzzahlen bei den Frauen und den anderen Altersgruppen praktisch unverändert.

Ähnlich stellt sich die Entwicklung des gegenwärtigen CannabisGebrauchs (Konsum innerhalb des letzten Monats) dar. Nachdem es in den 90er Jahren einen Anstieg des gegenwärtigen Konsums auf bis zu 10\% im Jahr 1997 gegeben hat, sind die Prävalenzraten 2001 wieder auf 5\% zurückgegangen. In 2004 lagen die Prävalenzraten des gegenwärtigen Cannabis-Gebrauchs ebenfalls bei 5\%. Auch der Anteil an „regelmäßigen“ Konsumen- ten (mehr als 10-mal in den letzten 12 Monaten) hat sich gegenüber 2001 (3\%) nicht geändert.

Schließlich ist das Durchschnittsalter des ersten Cannabis-Konsums (wie auch für andere illegale Drogen) in den letzten Jahren weitgehend stabil geblieben. In 1997 lag es bei 16,7, im Jahr 2001 bei 16,5 und in 2004 bei 16,4 Jahren.

In der Gesamtbetrachtung zeigen die Ergebnisse der neuesten Drogenaffinitätsstudie somit eine im Wesentlichen unveränderte Situation des Cannabis-Gebrauchs unter Jugendlichen in Deutschland innerhalb der letzten Jahre. Eine argumentative Grundlage zur Dramatisierung des so genannten Cannabis-Problems stellt diese Untersuchung nicht dar. Im Gegenteil: Insgesamt erhärtet sich der Eindruck, dass - im Gegensatz zur aktuellen öffentlichen und politischen Debatte um die vermeintliche Ausweitung des Cannabis-Konsums unter Jugendlichen sowie der damit verbundenen gesundheitlichen und sozialen Risiken und Probleme - die große Mehrheit der Jugendlichen und jungen Erwachsenen einen gesellschaftlich integrierten Konsum betreibt, wie er auch viele Jahre zuvor in Deutschland bestand. Cannabis-Gebrauch war und ist überwiegend Probier- oder sporadischer Konsum. Vielmehr sollte diese Studie herangezogen werden, um den Blick auf besondere Problemgruppen zu erhellen. Hier sollte insbesondere der Gruppe der männlichen 16- bis 19-Jährigen eine besondere Aufmerksamkeit zuteil werden, bei der sich ein leichter Trend der Zunahme des Cannabis-Konsums abzeichnet. Die Analyse und kritische Diskussion der sozialen Hintergründe und Konsummotive dieser Jugendlichen (sowie anderer Cannabis-Konsumenten, die ein riskantes Gebrauchsmuster entwickeln) und die Herausbildung adäquater Hilfsangebote bleibt eine wichtige Aufgabe.

\section{Repräsentativerhebung 2003}

Seit den 80er Jahren werden im Auftrag der Bundesregierung repräsentative Befragungen zum Konsum psychoaktiver Substanzen bei der erwachsenen Bevölkerung in Deutschland durchgeführt. Die Repräsentativerhebung 2003 („Epidemiologischer Suchtsurvey“) basiert auf den Angaben von über 8000 erwachsenen Personen im Alter von 18 bis 59 Jahren, die schriftlich befragt worden sind [7].

Nach den Ergebnissen dieser Befragung hat der Cannabis-Konsum in Deutschland in den letzten Jahren stark zugenommen, vor allem wird von einer zunehmenden Zahl junger CannabisKonsumenten berichtet. Danach ist in der Altersgruppe der 18bis 39-Jährigen der Wert für die 12-Monats-Prävalenz seit 1997 von $7 \%$ auf $12 \%$ (2003) angestiegen. ${ }^{3}$ Wird die Gruppe der (westdeutschen) 18 - bis 24 -Jährigen allein betrachtet, ergibt sich eine Zunahme von 13\% (1997) auf 23\% (2003). Der letztgenannte Befund passt jedoch nicht zu den Ergebnissen der BZgA-Studie, die für den gleichen Zeitraum keine Veränderung bei der 12-Mo-

\footnotetext{
3 Trendzahlen für die Gesamtgruppe der Befragten (18 bis 59 Jahre) werden nicht angegeben. Auch Trendangaben für die 30-Tages-Prävalenz werden nicht genannt.
} 
nats-Cannabis-Prävalenz der jungen Erwachsenen festgestellt hat (siehe oben). ${ }^{4}$

Das Gleiche gilt für das Einstiegsalter des Cannabis-Konsums: Während die Analysen der Repräsentativerhebung auf eine Verschiebung zu immer früheren Cannabis-Erfahrungen hindeuten, hat sich nach den Ergebnissen der BZgA-Studie das Durchschnittsalter des ersten Cannabis-Konsums seit 1997 nicht verändert (siehe oben).

In der Repräsentativerhebung wurde ferner mit Hilfe der Severityof-Dependence-Skala (SDS) versucht, den Anteil von cannabisabhängigen Personen zu bestimmen. Beim SDS wird in Abkehr vom biologischen Abhängigkeitsmodell über psychologische Komponenten der Schweregrad der Abhängigkeit operationalisiert. Die Skala besteht aus fünf Items, bei denen die Selbstauskünfte der Befragten so gewichtet werden, dass ein maximaler Wert von 15 Punkten erreicht werden kann. Zur Bestimmung der Kokain- oder Amphetaminabhängigkeit wurde von einigen Autoren ein Wert von drei oder mehr Punkten als Cut-off-Wert angenommen [8]. Bei Kokainabhängigen wurde die Validität von drei oder mehr Punkten in der SDS mit Gruppen mit und ohne Kokainabhängigkeit nach DSM-IV überprüft, bei Cannabis-Konsumenten jedoch bisher nicht [7]. Trotzdem wird dieser Schwellwert für Cannabis in der bundesdeutschen Repräsentativerhebung übernommen. Danach lassen sich 1,1\% der Befragten als cannabisabhängig diagnostizieren. Von den Cannabis-Konsumenten erreichen immerhin 16\% mindestens drei Punkte.

Dieses Verfahren ist jedoch methodisch fragwürdig: Der Cut-offWert für Kokain- und Amphetamine kann nämlich nicht einfach auf die weichere, anders wirkende Droge Cannabis übertragen werden. Im Ergebnis ist dadurch die Schwelle sehr niedrig, um eine Cannabis-Abhängigkeit zugeschrieben zu bekommen. Wenn man beispielsweise die Fragen „Wie häufig waren Sie über ihren Gebrauch von Cannabis besorgt“ und „Wie häufig haben Sie sich gewünscht, mit dem Konsum von Cannabis aufzuhören?“ mit der Antwortmöglichkeit „manchmal“ sowie die Frage „Wie schwierig fanden Sie es, mit dem Konsum Cannabis aufzuhören oder ganz auf Cannabis zu verzichten?" mit der Antwortmöglichkeit „etwas schwierig“ beantwortet, ist man nach dem gewählten Verfahren bereits eine cannabisabhängige Person. Ein solches Antwortverhalten dürfte zwar einen problematischen Konsum beschreiben, nicht jedoch psychische oder körperliche Abhängigkeit.

\section{Regionale Erhebungen 2003 und 2004}

\section{Schleswig-Holstein}

In Schleswig-Holstein wurde im Jahr 2004 eine repräsentative Prävalenzerhebung durchgeführt. Infratest dimap befragte 3500

\footnotetext{
4 In der Drogenaffinitätsstudie der BZgA wurden folgende Cannabis-Prävalenzen (12 Monate) für die Altersgruppe „20 bis 25 Jahre“ ermittelt: 15\% (1997), 14\% (2001), 15\% (2004) [6]. In den verschiedenen Prävalenzerhebungen wird fast immer mit unterschiedlichen Altersgruppierungen gearbeitet. Ein direkter Vergleich ist deshalb nur eingeschränkt möglich. Generelle Vergleichsbewertungen und Trendaussagen können jedoch auf einer solchen Basis getroffen werden.
}

Personen telefonisch zu ihrem Gesundheits- und Konsumverhalten. Danach gaben $8 \%$ der Jugendlichen (12 bis 25 Jahre) an, in den letzten 12 Monaten Cannabis konsumiert zu haben [10]. In der kurz davor veröffentlichten Drogenaffinitätsstudie der BZgA wurde - nach der gleichen Erhebungsmethode - ein Wert von $13 \%$ für diese Altersgruppe auf der Bundesebene ermittelt (siehe oben). Wird der Wert für die erwachsene Bevölkerung (18 bis 59 Jahre) betrachtet, zeigt sich, dass in Schleswig-Holstein $4 \%$ in den letzten 12 Monaten Cannabis-Produkte konsumiert haben. Der entsprechende Wert aus der Bundesstudie beträgt 7\% [7]. ${ }^{5}$ Ferner korrespondieren die ermittelten Prävalenzzahlen mit dem etwas niedrigeren Anteil von Cannabis-Klienten in der ambulanten Suchthilfe Schleswig-Holstein als bundesweit (siehe unten).

Die für Schleswig-Holstein festgestellten Prävalenzen sind vor allem deshalb interessant, weil in diesem Bundesland seit Jahren eine vergleichsweise liberale Regelung bei der Strafverfolgung von Cannabis-Delikten gilt. Danach ist bis zu einer Besitzmenge von 30 Gramm Cannabis zum Eigengebrauch ein Strafverfahren in der Regel einzustellen. Ferner herrscht seit Jahren in Schleswig-Holstein ein „mildes politisches Klima“, wenn es um die Cannabis-Problematik geht. Die meisten politischen Parteien setzen sich dort für eine Entkriminalisierung von Cannabis ein. Offensichtlich führt eine solche, liberalere Cannabis-Politik nicht zu einem erhöhten Cannabis-Konsum in der Bevölkerung.

\section{Hamburg}

Obwohl die Freie und Hansestadt Hamburg direkt an das Land Schleswig-Holstein angrenzt, lag die dort ermittelte 12-MonatsCannabis-Prävalenz für die erwachsene Bevölkerung (18 bis 59 Jahre) mit $12,5 \%$ dreimal so hoch. Das weist eine jüngst veröffentlichte spezielle Prävalenzerhebung für Hamburg aus [12].

Von denjenigen, die einen Cannabis-Konsum im letzten Jahr angaben, haben $25 \%$ einmal und $22 \%$ zwei- bis fünfmal konsumiert. Eine intensive Konsumhäufigkeit findet sich bei $22 \%$ der Cannabisgebraucher - sie haben häufiger als wöchentlich Cannabis-Produkte benutzt. Auch in der Hamburger Studie wurde methodisch mit der Severity-of-Dependence-Skala (SDS) gearbeitet (siehe oben). Danach wird ein Viertel der Konsumenten der letzten 12 Monate als cannabisabhängig eingestuft (das wären - nach den Hochrechnungen der Studie - 31200 Personen in Hamburg). Unklar ist allerdings, warum es bei einem Wert von $22 \%$ mit (mindestens) wöchentlichem Cannabis-Konsum eine Abhängigkeitsrate von 25 \% gibt, zumal nicht alle wöchentlichen Gebraucher die Kriterien einer Abhängigkeit erfüllen dürften.

In Hamburg hat sich der Cannabis-Konsum in den letzten Jahren stark verbreitet. So hat sich die Cannabis-Prävalenz (der letzten 12 Monate) zwischen 1997 und 2003 von 6,4\% auf 12,5\% fast ver-

5 Da diese geringere Cannabis-Prävalenz für Schleswig-Holstein mit dem empirischen Befund bei der Vergleichsauswertung der Jugendlichen übereinstimmt, kann davon ausgegangen werden, dass Verzerrungen durch den telefonischen Erhebungsmodus - ältere methodische Vergleichsstudien haben ergeben, dass es bei Telefoninterviews zu einer Unterschätzung der Cannabis-Prävalenz kommt [11] - eher geringer sind. Ferner wird dieser Prävalenzwert auch in einer Teilstichprobe für Schleswig-Holstein aus der bundesweiten Repräsentativerhebung mit $4,4 \%$ bestätigt. 
doppelt. ${ }^{6}$ Diese hohe Prävalenzrate bei den Erwachsenen korrespondiert mit derjenigen bei den Jugendlichen. Das zeigen empirische Ergebnisse einer regionalen Schülerbefragung für Hamburg [13]. Dort wird für das Jahr 2004 ein Wert von $31 \%$ bei den 15- bis 18-Jährigen angegeben, die in den letzten 12 Monaten zumindest einmal Cannabis konsumiert haben. ${ }^{7}$ Der Wert für die 30-Tages-Prävalenz lautet $19 \%$.

Es gibt also starke Unterschiede beim Cannabis-Konsum zwischen Hamburg und Schleswig-Holstein. Dies kann nicht allein mit dem bekannten Stadt-Land-Unterschied erklärt werden. Aus der Metropole Frankfurt sind Zahlen bekannt - wie gleich zu berichten sein wird -, nach denen der Cannabis-Konsum bei jungen Menschen sogar rückläufig ist.

\section{Frankfurt a.M.}

In der Stadt Frankfurt kommt seit einigen Jahren ein „Monitoring-System Drogentrends“ zur Anwendung. Mit ihm sollen epidemiologische Entwicklungen aufgezeigt und prognostische Aussagen getroffen werden. Methodisch besteht es aus einer Expertenbefragung, einer Schülerbefragung, einem „TrendscoutPanel" und einer Szenestudie [14]. Nach den Ergebnissen der Schülerbefragung (15 bis 18 Jahre, weiterführende und Berufschulen, $n=1500$ ) ist der Anteil von jugendlichen Cannabis-Konsumenten in den letzten 3 Jahren in Frankfurt zurückgegangen. Bei der 12-Monats-Prävalenz zeigt sich ein abnehmender Trend von 35\% (2002) über 32\% (2003) auf 24\% (2004). Die Werte für den Anteil aktueller Konsumenten (30-Tages-Prävalenz) lauten: $21 \%$ (2002), 19\% (2003) und 12\% (2004) [14].

Im Vergleich Frankfurt und Hamburg zeigt sich damit für das Jahr 2004 eine deutlich geringere Cannabis-Prävalenz für Frankfurt.

Die hier dargestellten regionalen Zahlen zeigen dreierlei:

1. Es gibt erhebliche regionale Unterschiede bei den CannabisPrävalenzen, wenn Hamburg, Frankfurt und Schleswig-Holstein miteinander verglichen werden.

2. Es ist nicht überall ein Anstieg des Cannabis-Konsums festzustellen (Frankfurt).

3. Offensichtlich führt eine moderate Praxis bei der Strafverfolgung nicht zu einem Anstieg des Cannabis-Konsums (Schleswig-Holstein).

Solche regionalen Erkenntnisse können die Diskussion über Entwicklungen beim Cannabis-Konsum in differenzierter Form beleben. Es sollten vertiefende und vergleichende Studien durchgeführt werden, in denen nicht nur Einstellungen und Konsummotive, sondern ebenfalls externe Bedingungen (CannabisMarkt, Präventionsangebote etc.) analysiert werden.

6 In dem Prävalenzwert für 1997 sind sogar die 15- bis 17-Jährigen mit enthalten.

7 Der Wert für die 12-Monats-Prävalenz findet sich nicht in dem Bericht; er wurde aber auf der Jahrestagung des Fachausschusses „Suchtprävention" (September 2004) in einer Präsentation vom Autor der Untersuchung, Theo Baumgärtner, genannt. Ferner hat er dankenswerterweise für diesen Beitrag die Zahlen für die Altersgruppe der 15- bis 18-jährigen Schüler berechnet, damit eine Vergleichbarkeit mit den Prävalenzen aus Frankfurt a. M. hergestellt werden kann.

\section{Deutsche Suchthilfestatistik 2003}

In der nationalen Suchthilfestatistik (früher: EBIS-Statistik) wird eine Zunahme des Anteils von cannabisbezogenen Störungen in den ambulanten Einrichtungen seit 1992 von 2,1\% auf 9,5\% im Jahr 2003 festgestellt $[9,15] .^{8}$ Die Steigerungsrate der letzten Jahre wird allgemein als weiterer Indikator für einen zunehmend problematischen Umgang mit Cannabis-Produkten - vor allem von jungen Menschen - angesehen.

Ohne die Tendenz der Zunahme von Behandlungsfällen wegen Cannabis an sich bestreiten zu wollen, ist jedoch fraglich, ob das Ausmaß wirklich behandlungsbedürftiger Fälle richtig erfasst wird. Durch die spezifische Erhebungslogik des Substanzkonsums in der deutschen Suchthilfestatistik dürfte es zu einer Überschätzung von behandlungsbedürftigen Cannabis-Klienten kommen. Diese werden nämlich in der deutschen Suchthilfestatistik seit Jahren ausschließlich über den ICD-10 erfasst.

Der ICD ist ein international anerkanntes Diagnoseschema zur Klassifikation somatischer und psychischer Störungen, für dessen Anwendung es spezielle Handbücher gibt [15]. Nur Personen, die über eine entsprechende psychologische oder psychiatrische Ausbildung verfügen oder speziell für den ICD-10 geschult worden sind, sollten mit diesem diagnostischen Instrument arbeiten. In den diagnostischen Leitlinien des ICD-10 ist beispielsweise die Empfehlung enthalten, Bestätigung aus mehreren Quellen zu suchen, bevor eine substanzbezogene Störung diagnostiziert wird.

In Wirklichkeit - dafür gibt es zahlreiche Hinweise aus der Praxis benutzen aber auch Mitarbeiter der Suchthilfe den ICD-10, die für die Anwendung dieses Instrumentes nie geschult worden sind. Deshalb ist es wahrscheinlich, dass es hierbei in einem nicht unerheblichen Ausmaß zu Fehldiagnosen kommt. Es sei angemerkt, dass hierbei kein individuelles Fehlverhalten vorliegt, sondern dass die Mitarbeiter dazu angehalten werden, aufgrund von Dokumentationsvorgaben mit dem ICD-10 den Substanzkonsum ihrer Klienten zu erfassen.

Das BMGS hat in einer von ihm in Auftrag gegebenen Studie die ICD-Diagnosestellung nachprüfen lassen [15]. Dabei wurde eine Quote von bis zu einem Viertel falsch vergebener Diagnosen festgestellt. Immerhin bedeutet dies, dass möglicherweise bis zu einem Viertel aller Cannabis-Klienten in den letzten Jahren falsch in der nationalen Suchthilfestatistik aufgetaucht sind. Bezogen auf die bundesdeutsche Suchtklientel dürfte dieser Anteil falsch diagnostizierter Cannabis-Konsumenten sogar noch höher liegen. In der Studie sind vor allem jene Klienten in die Stichprobe eingegangen, die im Mittel länger in Betreuung waren und häufiger Kontakte mit den Einrichtungen hatten als der Durchschnitt der Cannabis-Klienten. Bei den kürzer betreuten Cannabis-Klienten haben sich die Mitarbeiter noch kein vollständiges Bild über das Konsumverhalten und die potenziellen Störungen des Klienten gemacht, so dass die Möglichkeit einer Fehldiagnose hier noch größer ist.

\footnotetext{
8 Die Zahlen basieren auf den Angaben zu den Betreuungszugängen innerhalb eines Jahres.
} 
Es kann ferner vermutet werden, dass nicht nur der Anteil der Hauptdiagnosegruppe Cannabis falsch eingeschätzt wird, sondern auch das Verhältnis innerhalb der einzig möglichen Diagnoseoptionen „schädlicher Gebrauch“ oder „Abhängigkeit“ in der nationalen Suchthilfestatistik nicht stimmt. ${ }^{9}$ Dies ist vor allem darauf zurückzuführen, dass im Manual zum Kerndatensatz, auf dem die deutsche Suchthilfestatistik basiert, viele Jahre mit einer überholten diagnostischen Leitlinie gearbeitet worden ist, die die Anzahl cannabisabhängiger Personen künstlich erhöht haben dürfte. ${ }^{10}$

In der genannten Studie wird weiter festgestellt, dass die Anwendung der klinischen ICD-Diagnostik in den ambulanten Beratungsstellen seit 10 Jahren Standard ist. Wie schon ausgeführt, ist nach zahlreichen Kontakten mit Praktikern aus mehreren Bundesländern festzustellen, dass dies nicht der Fall ist. Vor allem im Bereich der Betreuung von Konsumenten illegaler Drogen sind vielfach Sozialarbeiter und Sozialpädagogen tätig, die weder für den ICD-10 geschult worden sind noch mit diesem Instrument arbeiten möchten - sei es aufgrund der inadäquaten Kategorisierung, seiner mangelnden Aussagekraft oder der unerwünschten Stigmatisierung des Konsumverhaltens junger Menschen. ${ }^{11}$

Bei 25\% aller in der bundesdeutschen Suchthilfestatistik 2003 erfassten Betreuungszugänge wurde keine Hauptdiagnose nach ICD-10 gestellt. ${ }^{12}$ Auch das ist ein Zeichen für Probleme, die im Zusammenhang mit der alleinigen Anwendung dieses diagnostischen Instrumentes entstehen. Denn entweder liegt bei dem betroffenen Personenkreis weder ein Abhängigkeitssyndrom noch ein schädliches Gebrauchsmuster vor, oder es gibt Mitarbeiter in der ambulanten Suchthilfe, die nicht dafür ausgebildet worden sind, mit dem ICD eine korrekte Diagnose zu stellen. In beiden Fällen fallen Personen, die eine ambulante Suchthilfeeinrichtung aufgesucht haben, durch das Dokumentationsraster und verzerren damit die Statistik.

In diesem Zusammenhang sei ausgeführt, dass sich im Bundesland Schleswig-Holstein, in dessen ambulanten Einrichtungen optional das Konsumverhalten der Klienten mit einfacheren Kategorien („Hauptdroge“) erfasst werden kann, in den letzten 3 Jahren der relative Anteil von Cannabis-Klienten, die eine Behandlung in einer ambulanten Suchthilfeeinrichtung beginnen, nicht erhöht hat (2001: 8\%, 2002: 7\%, 2003: 7\%) [18].

9 In der nationalen Suchthilfestatistik werden die Betreuungsfälle mit einer Diagnose „Abhängigkeitssyndrom“ oder einer Diagnose „schädlicher Gebrauch“ zur Hauptdiagnosegruppe „Cannabis“ zusammengefasst.

${ }^{10}$ So sollte ein Abhängigkeitssyndrom festgestellt werden, wenn während des letzten Jahres bei einem Klienten drei oder mehr von acht Kriterien gleichzeitig vorhanden waren. In Wirklichkeit werden in den ICD-Handbüchern seit vielen Jahren nur sechs Kriterien genannt zwei wurden als eigenständige Kategorien abgeschafft, weil sie eine starke inhaltliche Nähe zu anderen aufwiesen. Die Anwendung der alten, längst überholten Leitlinie erhöht damit prinzipiell die Wahrscheinlichkeit, eine Diagnose „Abhängigkeitssyndrom“ zu erhalten.

${ }^{11}$ So heißt es in einer Mitteilung des Hamburger Senats: „Einrichtungen der Drogenhilfe lehnen eine generelle Einführung des ICD-10 ab und weisen auf den hohen Qualifizierungsbedarf hin. Eine flächendeckende Anwendung sei nicht erforderlich“ [17].

12 Eigene Berechungen auf der Grundlage der Tabellenbände der nationalen Suchthilfestatistik (www.suchthilfestatistik.de).
Aus dem Genannten ergibt sich, dass der Substanzkonsum nicht mehr ausschließlich mit Hilfe des ICD-10 dokumentiert werden sollte. Es sollte daneben noch eine weitere Möglichkeit geben, z. B. die Erfassung der „Hauptsubstanz“ oder der 30-Tages-Prävalenz. Es kann erwartet werden, dass bei einem solchen optionalen Kategoriensystem validere Erkenntnisse zur Entwicklung und zum Ausmaß cannabisbezogener Störungen zustande kommen. Auf einer solchen, empirisch gesicherten Grundlage können dann fachliche und politische Diskussionen über mögliche Konsequenzen geführt werden.

Ein weiterer, wichtiger Punkt sei genannt, an dem die Zahlen zu cannabisbezogenen Störungen aus der nationalen Suchthilfestatistik kritisch reflektiert werden sollten: Jugendliche, die eine ambulante Suchthilfeeinrichtung aufsuchen, sind mehrheitlich Cannabis-Konsumenten, seltener Alkoholkonsumenten. Der schleswig-holsteinischen Suchthilfestatistik des Jahres 2002 ist zu entnehmen, dass es sich bei den 14- bis 17-jährigen Klienten zu 57\% um Personen mit der Hauptsubstanz Cannabis und nur zu 16\% um Personen mit der Hauptsubstanz Alkohol handelt [19]. ${ }^{13}$ Da nicht davon auszugehen ist, dass Jugendliche besser und kontrollierter mit Alkohol als mit Cannabis umgehen können und da ferner bekannt ist, dass das Abhängigkeitspotenzial von Cannabis zumindest nicht höher ist als das von Alkohol, dürften diese ungleichen Betreuungszahlen mit den Rahmenbedingungen des Konsums zusammenhängen. Unsere These ist: Besorgte Eltern, Lehrer oder Ausbilder schicken Konsumenten von Cannabis in die Beratungsstellen, nicht weil diese abhängig sind oder einen schädlichen Missbrauch ausüben, sondern weil es Ängste gibt, die im Zusammenhang mit dem Verbot von Cannabis stehen.

\section{Jahresbericht der Europäischen}

Beobachtungsstelle (EMCDDA)

Wie einleitend angemerkt, wird behauptet, dass überall in Europa der Cannabis-Konsum in den letzten Jahren zugenommen habe [2]. Das ist jedoch eine nicht zutreffende Aussage. Es stimmt zwar, dass sich in vielen Ländern die Prävalenzraten des Cannabis-Konsums erhöht haben, es gibt aber auch Staaten wie die Niederlande, Schweden, Finnland und Norwegen, in denen der Cannabis-Konsum in den letzten Jahren nicht weiter angestiegen ist. Das weist der letzte Jahresbericht (2004) der Europäischen Beobachtungsstelle für Drogen (EMCDDA) aus [20]. Beispielsweise sind bei den Amsterdamer Schülern seit Mitte der 90er Jahre die Cannabis-Prävalenzen (Lebenszeit und 30 Tage) sehr stabil [21]. Auch in der ESPAD-Studie, die auf den Angaben von 15- und 16-jährigen Schülern aus vielen europäischen Staaten basiert, wird nicht von einer einheitlichen Tendenz der $\mathrm{Zu}$ nahme des Cannabis-Konsums in Europa berichtet. Danach ist die Cannabis-Prävalenz (30 Tage) zwischen 1999 und 2003 nur

\footnotetext{
${ }^{13}$ Die Hauptdiagnosen-Anteile in der nationalen Suchthilfestatistik 2003 betragen:

15- bis 17-Jährige: $12 \%$ Alkohol und $64 \%$ Cannabis. 18- bis 19-Jährige: $16 \%$ Alkohol und $47 \%$ Cannabis. Eigene Berechungen auf der Grundlage der Tabellenbände der nationalen Suchthilfestatistik (www.suchthilfestatistik.de).
} 
in 3 Ländern (Großbritannien, Slowakei, Bulgarien) deutlich angestiegen. In 3 Ländern ist sie leicht gesunken, in 5 Ländern stabil geblieben und in 14 ist sie leicht angestiegen. ${ }^{14}$

In dem Jahres-Bericht der EMCDDA wird außerdem ausgeführt, dass es zumindest in zwei Ländern der EU in der letzten Zeit zu einer leichten Entpönalisierung des Cannabis-Konsums gekommen ist, obwohl dort eher höhere Konsumraten unter jungen Menschen vorliegen (Belgien, Großbritannien).

Dieser Sachverhalt weist wiederum darauf hin, dass die Diskussion um die Entkriminalisierung von Cannabis nicht ausschließlich vor dem Hintergrund tatsächlich oder vermeintlich wachsender Konsumentenzahlen geführt, sondern eine abgewogene Bewertung der intendierten und nicht intendierten Folgen des Cannabis-Verbotes vorgenommen werden sollte. Vergleichende Untersuchungen aus den Niederlanden und Deutschland zeigen, dass es trotz eines unterschiedlichen rechtlichen und politischen Umgangs mit Cannabis ähnliche Entwicklungen beim Konsum in beiden Ländern gibt [22, 23]. Auch wenn diese Studien schon etwas älter sind, können sie einen Beitrag für eine rationale Cannabis-Diskussion leisten. Denn offensichtlich sind andere Faktoren für den Cannabis-Konsum entscheidender als die generalpräventive Wirkung durch Strafandrohung.

\section{Schlussfolgerungen}

Werden die Ergebnisse aller hier vorgestellten nationalen Untersuchungen zusammen betrachtet, lässt sich zumindest der empirisch gesicherte Schluss ziehen, dass es in den letzten 10 Jahren zu keinem Rückgang des Cannabis-Konsums in Deutschland gekommen ist. Ob er hingegen zugenommen hat, und wenn ja in welchem Ausmaß, kann aufgrund der teilweise widersprüchlichen Ergebnisse der Untersuchungen nicht eindeutig festgestellt werden. Nach den ermittelten Prävalenzen der letzten BZgA-Studie ist der Cannabis-Konsum bei den jungen Erwachsenen seit 1997 stabil, nach den Ergebnissen der aktuellen Repräsentativerhebung hat er seither in dieser Altersgruppe zugenommen. Auch die Ergebnisse von regionalen Erhebungen lassen keinen eindeutigen Trend erkennen. Das Gleiche gilt für vorliegende Zahlen aus anderen europäischen Staaten.

Dieser Befund gilt selbst dann, wenn man aufgrund der unterschiedlichen Erhebungsmethodiken (schriftlich versus telefonisch) die einen Ergebnisse (Repräsentativerhebung) für zuverlässiger hält als die anderen (BZgA-Studie). Denn der telefonische Befragungsmodus kann zwar zu einer Unterschätzung der Cannabis-Prävalenz führen, dies berührt aber nicht die generelle Richtung des so ermittelten Trends.

Eine „Cannabis-Seuche“ - wie sie in einzelnen Veröffentlichungen und in Teilen der Fachöffentlichkeit behauptet wird - lässt sich also mit "nackten Zahlen“ nicht belegen. Aus Sicht der Autoren haben vielmehr eine selektive Ergebnisdarstellung, einseiti-

\footnotetext{
${ }^{14}$ Siehe hierzu die entsprechenden Informationen unter www.espad.org (ESPAD: The European School Survey Project on Alcohol and Other Drugs).
}

ge Interpretationen und der Einsatz teilweise kritikwürdiger methodischer Erhebungsinstrumente und Diagnosepraktiken zu einer öffentlichen Dramatisierung und einer damit verbundenen Pathologisierung des Cannabis-Konsums geführt.

Mit dieser Feststellung sollen nicht der Cannabis-Konsum bagatellisiert und Fälle schweren Missbrauches und individueller Problematik verleugnet werden, die ohne Zweifel gezielte Hilfen benötigen. Die Autoren sind aber der festen Überzeugung, dass nur eine empirisch angemessene, d.h. sachbezogene und differenzierte Problembeschreibung zu den richtigen fachlichen und politischen Konsequenzen führt. Überreaktionen und Dramatisierungen wenn diese zudem von Presse und Politik verstärkt werden - sind weder ein produktiver Beitrag für eine glaubwürdige Präventionspolitik noch einer rationalen Sucht- und Drogenpolitik förderlich. „Angst ist ein schlechter Ratgeber“, insbesondere wenn damit dem weit verbreiteten Positivimage einer Droge entgegengetreten werden soll. Es steht zu befürchten, dass die Warnbotschaften, derart verpackt, weniger die Konsumenten selbst erreichen, als deren Eltern, Erziehungsberechtigte, Lehrer und Ausbilder. In dieser Weise wird der Cannabis-Konsum seiner Thematisierbarkeit beraubt, d.h. mit zunehmender Problemzuschreibung werden Tabuisierungsprozesse in Gang gesetzt, die allen Präventionsstrategien zuwiderlaufen. Cannabis-Konsum wird potenziell zum Makel, gepaart mit der Illegalität zu einem Problemattribut, das im auf Vertrauensbildung angelegten Erziehungsprozess eine negative Funktion einnimmt: Jugendliche Cannabis-Konsumenten vertrauen sich aus Angst vor einer Störungs- oder zumindest Defizitzuschreibung und „Ausrufung“ eines Beratungs- und Behandlungswunsches den Eltern und Erziehern nicht an. Die Wichtigkeit von Peer-Groups gewinnt dadurch für jugendliche Konsumenten noch mehr an Bedeutung. Das ist zugegebenermaßen eine These, die empirisch noch nicht untersucht worden ist. Denn bislang existiert in Deutschland keine Forschung zu den Folgen und Wirkungen einer Verbotspolitik auf das Drogengebrauchsverhalten. Die Wirkungen der Cannabis-Dramatisierung lassen sich lediglich aus den Erfahrungen von Praktikern ableiten, die es vermehrt mit besorgten Eltern und Lehrern zu tun bekommen - sei es in Beratungsstellen oder Telefonhotlines.

Vor diesem Hintergrund ist dringend zu überprüfen, ob die diagnostischen Instrumente zur Bestimmung von Abhängigkeit wie der (im Beratungsalltag einzusetzende) ICD-10 oder die SDS ohne Einschränkung auf Cannabis-Konsumenten angewendet werden können. Die Gefahr scheint hier groß zu sein, Fehldiagnosen zu stellen und junge Menschen zu stigmatisieren.

Und schließlich ist eine Präventions-, Beratungs- und Behandlungspolitik zu forcieren, die auf der empirisch gesicherten Erkenntnis basiert, dass der Cannabis-Konsum im Wesentlichen ein passageres Phänomen der Adoleszenzphase ist. Daraus kann sich eine grundsätzliche Gelassenheit ergeben, die jedoch nicht mit „Wegschauen“ verwechselt werden darf, auf deren Grundlage weitere Präventionsangebote entwickelt werden können. Dabei sollte sich in der Zielformulierung der praktischen Intervention und Politik die bisher erfolgte Differenzierung der gesamten Sucht- und Drogenpolitik widerspiegeln: die Tatsache, dass sich in den letzten 20 Jahren neben der Abstinenzorientierung eine nicht mehr wegzudenkende schadensminimierende Perspektive etabliert hat, die einen eigenen Stellenwert besitzt 
[24]. Beide Zielperspektiven müssen auch im Umgang mit Cannabis beachtet und umgesetzt werden. Es ist dabei ebenfalls die individuelle Verantwortungsübernahme zu würdigen, also die Befähigung und das Empowerment als Anerkennung und Stärkung subjektiver Ressourcen und Fähigkeiten. Die Devise ist, den „Behandelten“ zum „Handelnden“ zu machen. Praktischmethodisch kann diese Orientierung die Wertschätzung und Integration individueller Expertise und Betroffenenkompetenz in den Beratungs- und Behandlungsprozess bedeuten (z. B. im Rahmen von Peer-support-Modellen). Konsequenterweise müssten dann auch die Themen Genuss und Funktion aufgegriffen werden, um zu einer zielgruppenspezifischen sowie lebensweltnahen Präventionsstrategie zu gelangen [25].

\section{Literatur}

${ }^{1}$ Themenschwerpunkt: Cannabis - Neue Erkenntnisse zu Grundlagen, Klinik und Therapie. Sucht. Zeitschrift für Wissenschaft und Praxis 2004; 6 (5)

${ }^{2}$ Bundesministerium für Gesundheit und Soziale Sicherung. „Jugendkult" Cannabis - Risiken werden oft verharmlost. Hilfen für riskante Konsumenten erforderlich. Pressemitteilung, 2004

${ }^{3}$ Bundeszentrale für gesundheitliche Aufklärung (BZgA). Cannabis bei Jugendlichen hoch im Kurs. Pressemitteilung, 2004

${ }^{4}$ CDU und SPD. In Verantwortung für Schleswig-Holstein: Arbeit, Bildung, Zukunft. Koalitionsvertrag für die 16. Legislaturperiode 2005 bis 2010. Kiel, 2005

${ }^{5}$ Gaßmann R. Deutsche Hauptstelle für Suchtfragen. Cannabis. Neue Beiträge zu einer alten Diskussion. Freiburg i.B: Lambertus, 2004

${ }^{6}$ Bundeszentrale für gesundheitliche Aufklärung (BZgA). Die Drogenaffinität Jugendlicher in der Bundesrepublik Deutschland. Wiederholungsbefragung 2004. Köln: BZgA, 2004

${ }^{7}$ Kraus L, Augustin R. Repräsentativerhebung zum Gebrauch und Missbrauch psychoaktiver Substanzen bei Erwachsenen in Deutschland. Epidemiologischer Suchtsurvey 2003. Sucht. Zeitschrift für Wissenschaft und Praxis 2005; (51) Sonderheft 1

${ }^{8}$ Kaye S, Darke S. Determing a diagnostic cut-off on the Severity of Dependence Scale (SDS) for cocaine dependence. Addiction 2002; 97: $727-731$

${ }^{9}$ Sonntag D, Welsch K. Deutsche Suchthilfestatistik 2003. Sucht. Zeitschrift für Wissenschaft und Praxis 2004; (50) Sonderheft 1

${ }^{10}$ Unveröffentlichter Forschungsbericht von Infratest dimap für das Ministerium für Soziales, Gesundheit und Verbraucherschutz des Landes Schleswig-Holstein. Kiel, 2005
${ }^{11}$ Kraus L, Bauernfeind R, Bühringer G. Epidemiologie des Drogenkonsums in Deutschland. Ergebnisse aus Bevölkerungssurveys 1990 bis 1996. Schriftenreihe des Bundesministeriums für Gesundheit. BadenBaden: Nomos, 1998: Band 107

${ }^{12}$ Kraus L, Augustin R, Orth B. Repräsentativerhebung zum Gebrauch und Missbrauch psychoaktiver Substanzen bei Erwachsenen in Hamburg. Epidemiologischer Suchtsurvey 2003. München, 2005: IFT-Berichte (Band Nr. 146)

${ }^{13}$ Baumgärtner T. Rauschmittelkonsumerfahrungen der Hamburger Jugendlichen und jungen Erwachsenen 2004. Zusammenfassender Basisbericht der Schüler- und Lehrerbefragung zum Umgang mit Suchtmitteln. Forschungsbericht. Hamburg, 2004

${ }^{14}$ Kemmesies UE, Werse B, Müller O et al. MoSyD-Jahresberichte 2003 und 2004 (im Druck). Drogentrends in Frankfurt am Main. Forschungsberichte 2004 und 2005

${ }^{15}$ Simon R, Sonntag D. Cannabisbezogene Störungen: Umfang, Behandlungsbedarf und Behandlungsangebot in Deutschland. Studie für das Bundesministerium für Gesundheit und soziale Sicherung. München, Forschungsbericht 2004

${ }^{16}$ Dilling H, Mombour W, Schmidt MH. Internationale Klassifikation psychischer Störungen. Bern/Göttingen/Toronto/Seattle: Verlag Hans Huber, 2005: ICD-10 Kapitel V (F)

${ }^{17}$ Bürgerschaft der Freien und Hansestadt Hamburg. Mitteilung des Senats an die Bürgerschaft. Ausstiegsorientierte Reform der Suchtkrankenhilfe in Hamburg. Hamburg, 2005: Drucksache 18/2202 vom 3.5.2005

${ }^{18}$ Schütze C, Kalke J, Buth S et al. Moderne Dokumentation in der ambulanten Suchtkrankenhilfe. Analysen zur Struktur des ambulanten Suchthilfesystems. Kiel: Eigenverlag, 2004: Band V

${ }^{19}$ Jugendliche und junge Erwachsene in der ambulanten Suchthilfe. Empirische Befunde vor dem Hintergrund des Kinder- und Jugendhilfegesetzes. Suchttherapie 2005 (6) 1: $20-27$

${ }^{20}$ Europäische Beobachtungsstelle für Drogen und Drogensucht (EMCCDA). Stand der Drogenproblematik in der Europäischen Union und in Norwegen. Jahresbericht 2004 Lissabon: Eigenverlag, 2004

${ }^{21}$ Van Laar M, van Gageldonk K, Ketelaars T et al. The Netherlands. Report on the drug situation 2003. Utrecht: Forschungsbericht, 2003

${ }^{22}$ Reuband KH. Drug use in Germany and the Netherlands. In: Waal H (Hrsg). Patterns on the European drug scene. An exploration of differences. Oslo: National Institute for Alcohol and Drug Research, 1998

${ }^{23}$ Reuband KH. Drogenkonsum und Drogenpolitik. Deutschland und die Niederlande im Vergleich. Opladen: Leske u. Budrich, 1992

${ }^{24}$ Ambros Uchtenhagen A. Geleitwort. In: Heudtlass JH, Stöver H (Hrsg). Risiko mindern. Frankfurt: Fachhochschulverlag, 2005

${ }^{25}$ Schneider W, Stöver H. Die Bedeutung des Konzeptes „Gesundheitsförderung“ für die Drogenhilfe - Einbezug von Betroffenenkompetenz und die Entwicklung von Drogenberatung. In: Heudtlass JH, Stöver H (Hrsg). Risiko mindern. Frankfurt: Fachhochschulverlag, 2005 